\title{
Seed germination reports for coastal sand dune species from Sicily
}

\begin{abstract}
Salmeri, C. \& Trubia, M.: Seed germination reports for coastal sand dune species from Sicily [In Magrini, S. \& Salmeri, C. (eds), Mediterranean plant germination reports - 1]. — Fl. Medit. 29: 277-287. 2019. https://doi.org/10.7320/FlMedit29.277

This study investigated seed germination in nine psammophilous species occurring in Sicilian sand dunes, some of which, such as Muscari gussonei, Launaea resedifolia and Pancratium maritimum, are endemic and/or rare and very scattered in the area. Different germination protocols were tested for one or more populations and the best germination results per species are provided, reporting experimental conditions and specific comments on the germination behaviour.
\end{abstract}

Key words: germination protocols, Mediterranean flora, psammophytes.

2. Centaurea sphaerocephala L. subsp. sphaerocephala (Asteraceae) (Fig. 1a)

\section{Accession data}

Si: $\quad$ Gela (Caltanissetta), Contrada Roccazzelle (WGS84: $37.093372^{\circ} \mathrm{N}, 14.165187^{\circ} \mathrm{E}$ ), $5 \mathrm{~m}$ a.sl., 08 Jun 2010, A. Lantieri \& R. Galesi (BGS-CT 32AL/NV/07, Banca del Germoplasma delle Specie Spontanee, Università di Catania).

Si: $\quad$ Sampieri (Ragusa), loc. Pineta (WGS84: $36.720606^{\circ} \mathrm{N}, 14.741661^{\circ} \mathrm{E}$ ), $2 \mathrm{~m}$ a.s.1., 11 Jul 2011, A. Lantieri \& R. Galesi (BGS-CT 01AL/RG/11, Banca del Germoplasma delle Specie Spontanee, Università di Catania).

\section{Germination data}

Pre-treatments: Manual removal of pappus. Disinfection with a $1 \%$ sodium hypochlorite $(\mathrm{NaClO})$ water solution for 5 minutes followed by 3 rinses in sterile distilled water.

Germination medium: 3 sheets of sterilized filter paper (Whatman 40), imbibed with $3 \mathrm{ml}$ of sterilized distilled water.

Sample size: 100 seeds for each test $(25 \times 4$ replicates $)$.

\section{Observations}

Germination tests carried out at different constant temperatures $\left(10^{\circ} \mathrm{C}\right.$ to $\left.25^{\circ} \mathrm{C}\right)$ indicated $20^{\circ} \mathrm{C}$ as optimal germination temperature, though significant interspecific variation was detected, with highly different germination rates between the two investigated populations. Samples from Roccazzelle, very close to the town of Gela, showed the highest germination 


\begin{tabular}{|c|c|c|c|c|c|c|c|}
\hline Germination & Thermoperiod & $\begin{array}{c}\text { Photoperiod } \\
{[\text { light/dark] }}\end{array}$ & $\mathbf{T}_{\mathbf{1}}[\mathbf{d}]$ & $\mathbf{T}_{\mathbf{5 0}}[\mathbf{d}]$ & $\mathbf{T}_{\max }[\mathbf{d}]$ & $\mathbf{M T G}[\mathbf{d}]$ & Accession code \\
\hline $\mathbf{9 7 . 0} \%$ & constant $20^{\circ} \mathrm{C}$ & $12 / 12 \mathrm{~h}$ & 2.0 & 3.4 & 22.0 & 5.0 & $\begin{array}{c}\text { BGS-CT } \\
32 \mathrm{AL} / \mathrm{NV} / 07\end{array}$ \\
\hline $\mathbf{8 3 . 0} \%$ & constant $20^{\circ} \mathrm{C}$ & $12 / 12 \mathrm{~h}$ & 2.0 & 4.3 & 31.0 & 7.9 & $\begin{array}{c}\mathrm{BGS}-\mathrm{CT} \\
01 \mathrm{AL} / \mathrm{RG} / 11\end{array}$ \\
\hline
\end{tabular}

percentages, all exceeding $87 \%\left(88 \%\right.$ at $10^{\circ} \mathrm{C}$ and $15^{\circ} \mathrm{C}, 97 \%$ at $20^{\circ} \mathrm{C}, 87 \%$ at $\left.25^{\circ} \mathrm{C}\right)$, while seed germination values ranged from $57 \%\left(10^{\circ} \mathrm{C}\right)$ to $81 \%\left(25^{\circ} \mathrm{C}\right)$ and $83 \%\left(20^{\circ} \mathrm{C}\right)$ in the other accession from Sampieri beach. Increase in temperature enhanced the germination speed by over $70 \%$ in the first accession $\left(\mathrm{T}_{50}=6.8\right.$ at $10^{\circ} \mathrm{C} v s \mathrm{~T}_{50}=1.8$ at $\left.25^{\circ} \mathrm{C}\right)$ and over $55 \%$ in the second one $\left(\mathrm{T}_{50}=8.8\right.$ at $10^{\circ} \mathrm{C} v s \mathrm{~T}_{50}=4$ at $\left.25^{\circ} \mathrm{C}\right)$. Temperature also affected the germination rate out of the optimal level of $20^{\circ} \mathrm{C}$, with lower values at both minor and major temperature for the two populations. Royal Botanic Gardens Kew (2019) reports for the species $82 \%$ germination under $20^{\circ} \mathrm{C}$ and light $8 / 16$ photoperiod.

\section{Cyperus capitatus Vand. (Cyperaceae) (Fig. 1b)}

\section{Accession data}

Si: Gela (Caltanissetta), Contrada Roccazzelle (WGS84: $37.093372^{\circ} \mathrm{N}, 14.165187^{\circ} \mathrm{E}$ ), $5 \mathrm{~m}$ a.sl., 08 Jun 2010, A. Lantieri \& R. Galesi (BGS-CT 07AL/RG/10, Banca del Germoplasma delle Specie Spontanee, Università di Catania).

\section{Germination data}

Pre-treatments: Disinfection with a $1 \%$ sodium hypochlorite $(\mathrm{NaClO})$ water solution for 5 minutes followed by 3 rinses in sterile distilled water.

Germination medium: 3 sheets of sterilized filter paper (Whatman 40), imbibed with $3 \mathrm{ml}$ of sterilized distilled water.

Sample size: 100 seeds for each test $(25 \times 4$ replicates $)$.

\section{Observations}

Based on Redondo-Gómez \& al. (2011), C. capitatus seeds from SW Spain exhibit their greatest germination values at salinity levels between 0 and $1 \%$ under a $16 \mathrm{~h} \mathrm{light/8}$ $\mathrm{h}$ dark photoperiod and $25 / 15^{\circ} \mathrm{C}$ thermoperiod. Salt concentration $>1 \%$ in the substrate completely inhibited seed germination. Germination results, here firstly given for Sicilian plants, agree with these data since seeds achieved the highest germination percentage in distilled water, as well as in $0.1 \mathrm{M} \mathrm{NaCl}$ water solution though with much longer germination time. Conversely, increasing $\mathrm{NaCl}$ concentration $(0.2 \mathrm{M}, 0.3 \mathrm{M}, 0.5 \mathrm{M})$ in the germination medium strongly affected seed germination up to total inhibition $(\mathrm{G}=0 \%)$ at the higher salinity conditions. Seed germination under light conditions was just a bit lower than in full darkness, indicating that seeds are not affected by photoinhibition and light 


\begin{tabular}{|c|c|c|c|c|c|c|}
\hline Germination & Thermoperiod & $\begin{array}{c}\text { Photoperiod } \\
{[\text { light/dark] }}\end{array}$ & $\mathbf{T}_{\mathbf{1}}$ [d] & $\mathbf{T}_{\mathbf{5 0}}$ [d] & $\mathbf{T}_{\mathbf{m a x}}$ [d] & MTG [d] \\
\hline $\mathbf{1 0 0 \%}$ & $25 / 15^{\circ} \mathrm{C}$ & $0 / 24 \mathrm{~h}$ & 7.0 & 7.7 & 22.0 & 10.3 \\
\hline $\mathbf{9 6 . 7 \%}$ & $25 / 15^{\circ} \mathrm{C}$ & $12 / 12 \mathrm{~h}$ & 13.0 & 15.3 & 28.0 & 16.7 \\
\hline $\mathbf{9 6 . 7 \% ( \mathbf { 1 }}$ & $25 / 15^{\circ} \mathrm{C}$ & $0 / 24 \mathrm{~h}$ & 8.0 & 13.5 & 31.0 & 15.3 \\
\hline $\mathbf{9 1 . 7 \%}^{(\mathbf{1})}$ & $25 / 15^{\circ} \mathrm{C}$ & $12 / 12 \mathrm{~h}$ & 16.0 & 25.9 & 35.0 & 27.2 \\
\hline
\end{tabular}

(1) $0.1 \mathrm{M} \mathrm{NaCl}$ concentration in the germination medium.

only reduces the germination rate $(\mathrm{Pi}<0.1$, Carta $\&$ al. 2017). Alternating temperature also was a determinant factor for germination success, as germination tests carried out on this accession under constant temperatures $\left(10^{\circ} \mathrm{C}, 15^{\circ} \mathrm{C}, 20^{\circ} \mathrm{C}, 25^{\circ} \mathrm{C}\right)$ showed very low germination values, ranging from $0 \%$ to $17 \%$, except for $25^{\circ} \mathrm{C}$ with $57 \%$. Constant temperatures appeared to increase photoinhibition since germination values in light-incubated seeds $(12 / 12 \mathrm{~h}$ ) did not exceed 15\%, with photoinhibition index (Pi) ranging between 0.7 and 1 that indicates strong inhibitory effects of light (Carta \& al. 2017). Contrarily, Royal Botanic Gardens Kew (2019) reports $80 \%$ of germination at a constant temperature of $31^{\circ} \mathrm{C}$ and $12 / 12 \mathrm{~h}$ photoperiod, though only 20 seeds were tested.

4. Launaea fragilis (Asso) Pau (Asteraceae) (Fig. 1c)

\section{Accession data}

Si: $\quad$ Sampieri (Ragusa), loc. Pineta (WGS84: $\left.36.720606^{\circ} \mathrm{N}, 14.741661^{\circ} \mathrm{E}\right), 2 \mathrm{~m}$ a.s.1., 11 Jul 2011, A. Lantieri \& R. Galesi (BGS-CT 02AL/RG/11, Banca del Germoplasma delle Specie Spontanee, Università di Catania).

\section{Germination data}

Pre-treatments: Manual removal of pappus. Disinfection with a $1 \%$ sodium hypochlorite $(\mathrm{NaClO})$ water solution for 5 minutes followed by 3 rinses in sterile distilled water.

Germination medium: 3 sheets of sterilized filter paper (Whatman 40), imbibed with $3 \mathrm{ml}$ of sterilized distilled water.

Sample size: 100 seeds for each test $(25 \times 4$ replicates $)$. 


\begin{tabular}{|c|c|c|c|c|c|c|}
\hline Germination & Thermoperiod & $\begin{array}{c}\text { Photoperiod } \\
{[\text { light/dark] }}\end{array}$ & $\mathbf{T}_{\mathbf{1}}[\mathbf{d}]$ & $\mathbf{T}_{\mathbf{5 0}}$ [d] & $\mathbf{T}_{\max }[\mathbf{d}]$ & MTG [d] \\
\hline $\mathbf{9 9 . 0 \%}$ & constant $25^{\circ} \mathrm{C}$ & $12 / 12 \mathrm{~h}$ & 2.0 & 3.6 & 15.0 & 4.9 \\
\hline $\mathbf{9 8 . 0} \%$ & constant $15^{\circ} \mathrm{C}$ & $12 / 12 \mathrm{~h}$ & 2.0 & 11.2 & 31.0 & 12.4 \\
\hline
\end{tabular}

\section{Observations}

This species is a suffruticous chamaephyte with a Saharo-Sindian distribution area, which in Italy only occurs in the sand dunes of the southern and south-eastern Sicily, where it is rather rare and grows in the Ammophila arenaria mobile dune community. This is the first germination report for the species. Germination tests carried out on fresh seeds provided successful germination results also at $20^{\circ} \mathrm{C}(90 \%)$, while only $70 \%$ of germination was reached at $10^{\circ} \mathrm{C}$.

\section{Medicago marina L. (Fabaceae) (Fig. 1d)}

\section{Accession data}

Si: $\quad$ Sampieri (Ragusa), loc. Pineta (WGS84: $\left.36.720606^{\circ} \mathrm{N}, 14.741661^{\circ} \mathrm{E}\right), 2 \mathrm{~m}$ a.s.l., 11 Jul 2011, A. Lantieri \& R. Galesi (BGS-CT 03AL/RG/11, Banca del Germoplasma delle Specie Spontanee, Università di Catania).

\section{Germination data}

Pre-treatments: Disinfection with a $1 \%$ sodium hypochlorite $(\mathrm{NaClO})$ water solution for 5 minutes followed by 3 rinses in sterile distilled water. Mechanical scarification by sandpaper for 5 minutes. Imbibition in distilled water for $24 \mathrm{~h}$.

Germination medium: 3 sheets of sterilized filter paper (Whatman 40) imbibed with $3 \mathrm{ml}$ of sterilized distilled water.

Sample size: 100 seeds for each test $(25 \times 4$ replicates $)$.

\begin{tabular}{|c|c|c|c|c|c|c|}
\hline Germination & Thermoperiod & $\begin{array}{c}\text { Photoperiod } \\
{[\text { light/dark] }}\end{array}$ & $\mathbf{T}_{\mathbf{1}}$ [d] & $\mathbf{T}_{\mathbf{5 0}}$ [d] & $\mathbf{T}_{\text {max }}$ [d] & MTG [d] \\
\hline $\mathbf{9 8 . 0} \%$ & constant $10^{\circ} \mathrm{C}$ & $12 / 12 \mathrm{~h}$ & 3.0 & 4.4 & 22.0 & 6.1 \\
\hline $\mathbf{9 8 . 0} \%$ & constant $15^{\circ} \mathrm{C}$ & $12 / 12 \mathrm{~h}$ & 2.0 & 2.7 & 22.0 & 4.4 \\
\hline $\mathbf{9 8 . 0} \%$ & constant $20^{\circ} \mathrm{C}$ & $12 / 12 \mathrm{~h}$ & 1.0 & 2.1 & 13.0 & 3.1 \\
\hline
\end{tabular}




\section{Observations}

The examined Sicilian population of Medicago marina revealed an optimal temperature range of $10-20^{\circ} \mathrm{C}$, which agree with Royal Botanic Gardens Kew (2019), where $100 \%$ germination was reported at both $15^{\circ} \mathrm{C}$ and $20^{\circ} \mathrm{C}$ with $12 / 12$ photoperiod. Lower germination percentage $(89 \%)$ was achieved at $25^{\circ} \mathrm{C}$ with the same pre-treatments. Seeds resulted positively photoblastic, because germination tests under full darkness condition provided a bit lower results, with seed germination percentages ranging from $87 \%$ to $90 \%$ at the same temperature regimes as the light-incubated seeds. Again, increase in temperature $\left(25^{\circ} \mathrm{C}\right)$ led to lower seed germination percentage (50\%), which is in contrast with the $98 \%$ germination obtained by Scippa \& al. (2011) for plants from the Molise coastal dunes (Centralsouth Italy) under the same conditions. Ballesteros \& al. (2015) reported a germination percentage of $95 \%$ for dark-incubated seeds at $20^{\circ} \mathrm{C}$, but previously chemically scarified with sulfuric acid for 20 minutes.

\section{Muscari gussonei (Parl.) Nyman (Asparagaceae) (Fig. 1e)}

\section{Accession data}

Si: $\quad$ Gela (Caltanissetta), Contrada Roccazzelle (WGS84: $37.095833^{\circ} \mathrm{N}, 14.161388^{\circ} \mathrm{E}$ ), $3 \mathrm{~m}$ a.s.1., 08 Jun 2010, A. Lantieri \& R. Galesi (BGS-CT 06AL/RG/10, Banca del Germoplasma delle Specie Spontanee, Università di Catania).

\section{Germination data}

Pre-treatments: Disinfection with a $1 \%$ sodium hypochlorite $(\mathrm{NaClO})$ water solution for 5 minutes followed by 3 rinses in sterile distilled water.

Germination medium: 3 sheets of sterilized filter paper (Whatman 40) imbibed with $3 \mathrm{ml}$ of sterilized distilled water.

Sample size: 100 seeds for each test $(25 \times 4$ replicates $)$.

\begin{tabular}{|c|c|c|c|c|c|c|}
\hline Germination & Thermoperiod & $\begin{array}{c}\text { Photoperiod } \\
{[\text { light/dark] }}\end{array}$ & $\mathbf{T}_{\mathbf{1}}[\mathbf{d}]$ & $\mathbf{T}_{\mathbf{5 0}}$ [d] & $\mathbf{T}_{\max }$ [d] & MTG [d] \\
\hline $\mathbf{1 0 0} \%$ & constant $10^{\circ} \mathrm{C}$ & $0 / 24 \mathrm{~h}$ & 11.0 & 13.4 & 35.0 & 15.4 \\
\hline $\mathbf{9 8 . 0} \%$ & constant $15^{\circ} \mathrm{C}$ & $12 / 12 \mathrm{~h}$ & 12.0 & 13.7 & 36.0 & 14.9 \\
\hline
\end{tabular}

\section{Observations}

Muscari gussonei is an Endangered endemic species (Orsenigo \& al. 2018) which occurs in small fragmented populations along the south-western coast of Sicily. Seed germination resulted negatively affected by temperature since it decreased with increasing 
temperature $\left(84 \%\right.$ at $20^{\circ} \mathrm{C}, 0 \%$ at $\left.25^{\circ} \mathrm{C}\right)$. No statistically significant effect of light was instead detected among germination percentages, which were high and similar for both light- and dark-incubated seeds under $10^{\circ} \mathrm{C}(100 \%$, in accordance with Royal Botanic Gardens Kew 2019) and $15^{\circ} \mathrm{C}(97 \%)$.

\section{Ononis variegata L. (Fabaceae) (Fig. 1f)}

\section{Accession data}

Si: $\quad$ Gela (Caltanissetta), Contrada Roccazzelle (WGS84: $37.093372^{\circ} \mathrm{N}, 14.165187^{\circ} \mathrm{E}$ ), $5 \mathrm{~m}$ a.sl., $23 \mathrm{Jul}$ 2007, A. Lantieri \& R. Galesi (BGS-CT 32AL/NV/07, Banca del Germoplasma delle Specie Spontanee, Università di Catania).

Si: Riserva Naturale Orientata Oasi del Simeto (Catania), foce del fiume Simeto

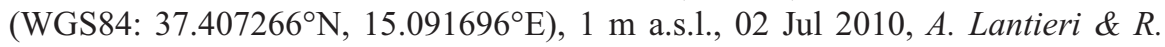
Galesi (BGS-CT 11AL/RG/10, Banca del Germoplasma delle Specie Spontanee, Università di Catania).

\section{Germination data}

Pre-treatments: Disinfection with a $1 \%$ sodium hypochlorite $(\mathrm{NaClO})$ water solution for 5 minutes followed by 3 rinses in sterile distilled water. Mechanical scarification by sandpaper for 5 minutes. Imbibition in distilled water for $24 \mathrm{~h}$.

Germination medium: 3 sheets of sterilized filter paper (Whatman 40), imbibed with $3 \mathrm{ml}$ of sterilized distilled water.

Sample size: 100 seeds for each test $(25 \times 4$ replicates $)$.

\begin{tabular}{|c|c|c|c|c|c|c|}
\hline Germination & Thermoperiod & $\begin{array}{c}\text { Photoperiod } \\
\text { [light/dark] }\end{array}$ & $\mathbf{T}_{\mathbf{1}}$ [d] & $\mathbf{T}_{\mathbf{5 0}}$ [d] & $\mathbf{T}_{\text {max }}$ [d] & MTG [d] \\
\hline $\mathbf{1 0 0 \%}$ & constant $10^{\circ} \mathrm{C}$ & $12 / 12 \mathrm{~h}$ & 2.0 & 2.3 & 8.0 & 3.3 \\
\hline $\mathbf{1 0 0} \%$ & constant $15^{\circ} \mathrm{C}$ & $12 / 12 \mathrm{~h}$ & 1.0 & 2.1 & 7.0 & 3.2 \\
\hline $\mathbf{9 9 . 0 \%}$ & constant $20^{\circ} \mathrm{C}$ & $12 / 12 \mathrm{~h}$ & 1.0 & 1.3 & 12.0 & 2.0 \\
\hline
\end{tabular}

\section{Observations}

The species shows a physical dormancy linked to its hard, impermeable seed coat, which needs scarification pre-treatments. The examined Sicilian populations of Ononis variegata revealed a wide range of optimal germination temperature from $10^{\circ} \mathrm{C}$ to $25^{\circ} \mathrm{C}$, with maximum germination percentages very close to $100 \%$. These results to some extent agree with existing data from Royal Botanic Gardens Kew (2019). Germination tests carried out under $24 \mathrm{~h}$ dark photoperiod provided germination rates similar to those of light-incubated seeds 
(98-100\%), but with faster germination speed $\left(\mathrm{T}_{50}=1-1.1 \mathrm{~d}, \mathrm{MTG}=2.1-2.2 \mathrm{~d}\right)$. The population from the coastal area of Simeto River showed a very similar germination behaviour, i.e. $97-99 \%$ under $12 / 12 \mathrm{~h}$ photoperiod and $97-100 \%$ under $24 \mathrm{~h}$ dark photoperiod, at constant temperature regimes from $10^{\circ} \mathrm{C}$ to $25^{\circ} \mathrm{C}$, but germination speed was much faster with lower values of $\mathrm{T}_{50}$ and $\mathrm{MTG}$.

8. Pancratium maritimum L. (Amaryllidaceae) (Fig. 1g)

\section{Accession data}

Si: Marsala (Trapani), Isola Grande dello Stagnone (WGS84: $37.903868^{\circ} \mathrm{N}$, 12.455125 ${ }^{\circ}$ ), $1 \mathrm{~m}$ a.s.1., 02 Oct 2004, S. Pasta \& L. Scuderi (BGS-CT 087G5/04, Banca del Germoplasma delle Specie Spontanee, Università di Catania).

Si: Gela (Caltanissetta), Spiaggia di Macchitella (WGS84: $37.076439^{\circ} \mathrm{N}$, 14.210627 ${ }^{\circ}$ E), 6 m a.s.1., 26 Sept 2004, Lantieri, Sciandrello \& Visalli (BGS-CT 052G1/04, Banca del Germoplasma delle Specie Spontanee, Università di Catania).

Si: Riserva Naturale Orientata Oasi del Simeto (Catania), foce del fiume Simeto (WGS84: $37.407266^{\circ} \mathrm{N}, 15.091696^{\circ} \mathrm{E}$ ), 28 Sept 2005, Restuccia \& Visalli (BGSCT 060AR/NV/05, Banca del Germoplasma delle Specie Spontanee, Università di Catania).

\section{Germination data}

Pre-treatments: Disinfection with a $1 \%$ sodium hypochlorite $(\mathrm{NaClO})$ water solution for 5 minutes followed by 3 rinses in sterile distilled water.

Germination medium: 3 sheets of sterilized filter paper (Whatman 40), imbibed with $3 \mathrm{ml}$ of sterilized distilled water.

Sample size: 100 seeds for each test $(25 \times 4$ replicates $)$.

\begin{tabular}{|c|c|c|c|c|c|c|c|}
\hline Germination & Thermoperiod & $\begin{array}{c}\text { Photoperiod } \\
{[\text { light/dark] }}\end{array}$ & $\mathbf{T}_{\mathbf{1}}[\mathbf{d}]$ & $\mathbf{T}_{\mathbf{5 0}}[\mathbf{d}]$ & $\mathbf{T}_{\max }[\mathbf{d}]$ & $\mathbf{M T G}_{\text {[d] }}$ & Accession code \\
\hline $\mathbf{9 8 . 0} \%$ & constant $20^{\circ} \mathrm{C}$ & $12 / 12 \mathrm{~h}$ & 1.0 & 6.7 & 14.0 & 7.2 & $\begin{array}{c}\text { BGS-CT } \\
087 \mathrm{G} 5 / 04\end{array}$ \\
\hline $\mathbf{9 8 . 0} \%$ & constant $20^{\circ} \mathrm{C}$ & $12 / 12 \mathrm{~h}$ & 5.0 & 10.0 & 25.0 & 11.0 & $\begin{array}{c}\text { BGS-CT } \\
052 \mathrm{G} 1 / 04\end{array}$ \\
\hline $\mathbf{9 8 . 0} \%$ & constant $20^{\circ} \mathrm{C}$ & $12 / 12 \mathrm{~h}$ & 3.0 & 7.7 & 18.0 & 9.0 & $\begin{array}{c}\text { BGS-CT } \\
060 \mathrm{AR} / \mathrm{NV} / 05\end{array}$ \\
\hline
\end{tabular}

\section{Observations}

Three different populations of $P$. maritimum from Sicily were investigated, coming from 3 opposite areas, W Sicily (Isola Grande), S Sicily (Gela) and E Sicily (Simeto river), which are characterized by the same mean annual temperature (around $17.5^{\circ} \mathrm{C}$ ), but by dif- 
ferent mean annual rainfall and relative humidity $(448.6 \mathrm{~mm} / 77.5 \%, 354.2 \mathrm{~mm} / 75.6 \%$, and $547.2 \mathrm{~mm} / 69.9 \%$, respectively). However, each accession had its optimal germination response $(98 \%)$ at a constant temperature of $20^{\circ} \mathrm{C}$ with $12 / 12$ photoperiod. High levels of germination, all exceeding $92 \%$, were also obtained at other constant temperatures: $92-$ $95 \%$ at $10^{\circ} \mathrm{C}, 93-96 \%$ at $15^{\circ} \mathrm{C}, 92-97 \%$ at $25^{\circ} \mathrm{C}$. Increasing temperatures in general enhanced seed germination speed $\left(\mathrm{T}_{50}\right.$ of c. $25 \mathrm{~d}$ at $10^{\circ} \mathrm{C}, \mathrm{c} .17 \mathrm{~d}$ at $15^{\circ} \mathrm{C}$, and c. $10 \mathrm{~d}$ at $15^{\circ} \mathrm{C}$ ). Seeds from all populations resulted positively photoblastic, because germination tests under full darkness provided very low germination rates at each thermoperiod, not exceeding $50 \%$, which is in contrast with germination results from Sardinian and Tuscan populations (Bacchetta \& al. 2007; Balestri \& Cinelli 2004).

9. Plantago macrorrhyza Poir. (Plantaginaceae) (Fig. 1h)

\section{Accession data}

Si: $\quad$ Sampieri (Ragusa), loc. Pineta (WGS84: $\left.36.720606^{\circ} \mathrm{N}, 14.741661^{\circ} \mathrm{E}\right), 2 \mathrm{~m}$ a.s.1., 11 Jul 2011, A. Lantieri \& R. Galesi (BGS-CT 04AL/RG/11, Banca del Germoplasma delle Specie Spontanee, Università di Catania).

\section{Germination data}

Pre-treatments: Disinfection with a $1 \%$ sodium hypochlorite $(\mathrm{NaClO})$ water solution for 5 minutes followed by 3 rinses in sterile distilled water.

Germination medium: 3 sheets of sterilized filter paper (Whatman 40), imbibed with $3 \mathrm{ml}$ of sterilized distilled water.

Sample size: 100 seeds for each test $(25 \times 4$ replicates $)$.

\begin{tabular}{|c|c|c|c|c|c|c|}
\hline Germination & Thermoperiod & $\begin{array}{c}\text { Photoperiod } \\
{[\text { light/dark] }}\end{array}$ & $\mathbf{T}_{\mathbf{1}}$ [d] & $\mathbf{T}_{\mathbf{5 0}}$ [d] & $\mathbf{T}_{\text {max }}$ [d] & MTG [d] \\
\hline $\mathbf{1 0 0 \%}$ & constant $10^{\circ} \mathrm{C}$ & $12 / 12 \mathrm{~h}$ & 2.0 & 3.7 & 17.0 & 5.2 \\
\hline $\mathbf{1 0 0} \%$ & constant $15^{\circ} \mathrm{C}$ & $12 / 12 \mathrm{~h}$ & 1.0 & 2.3 & 9.0 & 2.9 \\
\hline $\mathbf{1 0 0} \%$ & constant $20^{\circ} \mathrm{C}$ & $12 / 12 \mathrm{~h}$ & 1.0 & 2.3 & 6.0 & 2.6 \\
\hline
\end{tabular}

\section{Observations}

The species shows a moderate sensitivity to temperature and salinity. Temperature regimes over $25^{\circ} \mathrm{C}$ tended to induce seed dormancy, with delay in both germination time and final germination percentage $\left(94 \%\right.$ at $25^{\circ} \mathrm{C}, 50 \%$ at $\left.30^{\circ} \mathrm{C}\right)$; a $50 \%$ germination is also reported at $25^{\circ} \mathrm{C}$ and $12 / 12 \mathrm{~h}$ photoperiod by Royal Botanic Gardens Kew (2019). These results are in accordance with those given by Luciani \& al. (2001), who studied germina- 
tion behaviour of this species related to temperature, salinity and after-ripening time, assessing an optimal germinability $(98 \%)$ at $15^{\circ} \mathrm{C}$ (full darkness and distilled water), and a seed dormancy induction with both rising temperature $\left(>20^{\circ} \mathrm{C}\right)$ and salinity $(>0.1 \mathrm{M}$ $\mathrm{NaCl}$ ), but basically overcome by longer after-ripening time.

10. Thinopyrum junceum (L.) Á. Löve (Poaceae) (Fig. 1i)

\section{Accession data}

Si: Riserva Naturale Orientata Oasi del Simeto (Catania), foce del fiume Simeto (WGS84: $37.407266^{\circ} \mathrm{N}, 15.091696^{\circ} \mathrm{E}$ ), $2 \mathrm{~m}$ a.s.1., 09 Aug 2010, A. Lantieri \& $R$. Galesi (BGS-CT 07AL/RG/10, Banca del Germoplasma delle Specie Spontanee, Università di Catania).

\section{Germination data}

Pre-treatments: Disinfection with a $1 \%$ sodium hypochlorite $(\mathrm{NaClO})$ water solution for 5 minutes followed by 3 rinses in sterile distilled water.

Germination medium: 3 sheets of sterilized filter paper (Whatman 40), imbibed with $3 \mathrm{ml}$ of sterilized distilled water.

Sample size: 100 seeds for each test $(25 \times 4$ replicates $)$.

\begin{tabular}{|c|c|c|c|c|c|c|}
\hline Germination & Thermoperiod & $\begin{array}{c}\text { Photoperiod } \\
{[\text { light/dark] }}\end{array}$ & $\mathbf{T}_{\mathbf{1}}[\mathbf{d}]$ & $\mathbf{T}_{\mathbf{5 0}}[\mathbf{d}]$ & $\mathbf{T}_{\mathbf{m a x}}[\mathbf{d}]$ & $\mathbf{M T G}[\mathbf{d}]$ \\
\hline $\mathbf{7 8 . 3 \%}$ & $25 / 15^{\circ} \mathrm{C}$ & $12 / 12 \mathrm{~h}$ & 6.0 & 7.9 & 23.0 & 9.8 \\
\hline $\mathbf{7 3 . 3 \%}$ & $25 / 15^{\circ} \mathrm{C}$ & $0 / 24 \mathrm{~h}$ & 6.0 & 4.9 & 16.0 & 6.8 \\
\hline
\end{tabular}

\section{Observations}

Results of germination tests less than $80 \%$ were due to the persistency of embedded seeds which did not show coat rupture and radicle protrusion. Since these seeds included a vital embryo, mechanical strengths by the pericarp are plausible (Mavroeidi 2015). Tests performed with the addition of $\mathrm{NaCl}$ water solutions to the germination medium revealed that seed germination of Sicilian plants is negatively affected by salinity. In fact, while low $\mathrm{NaCl}$ concentration $(0.1 \mathrm{M})$ did not alter significantly the germination rate $(\mathrm{G}=72-75 \%)$, increasing salt quantities in the germination medium progressively delayed germination, increased MTG and reduced final germination $(65 \%$ with $0.2 \mathrm{M} \mathrm{NaCl}, 52 \%$ with $0.3 \mathrm{M}$, and $30 \%$ with $0.5 \mathrm{M}$ ). Light had no significant effects on final germination; tests carried out under $12 / 12 \mathrm{~h}$ photoperiod provided comparable results to full darkness (from $78.3 \%$ without salt to $72 \%$ with $0.1 \mathrm{M} \mathrm{NaCl}$ or less with higher salt concentrations). Ballesteros \& al. (2015) reported about $90 \%$ of germination with a $12 / 12 \mathrm{~h}$ photoperiod and $20^{\circ} \mathrm{C}$ constant 


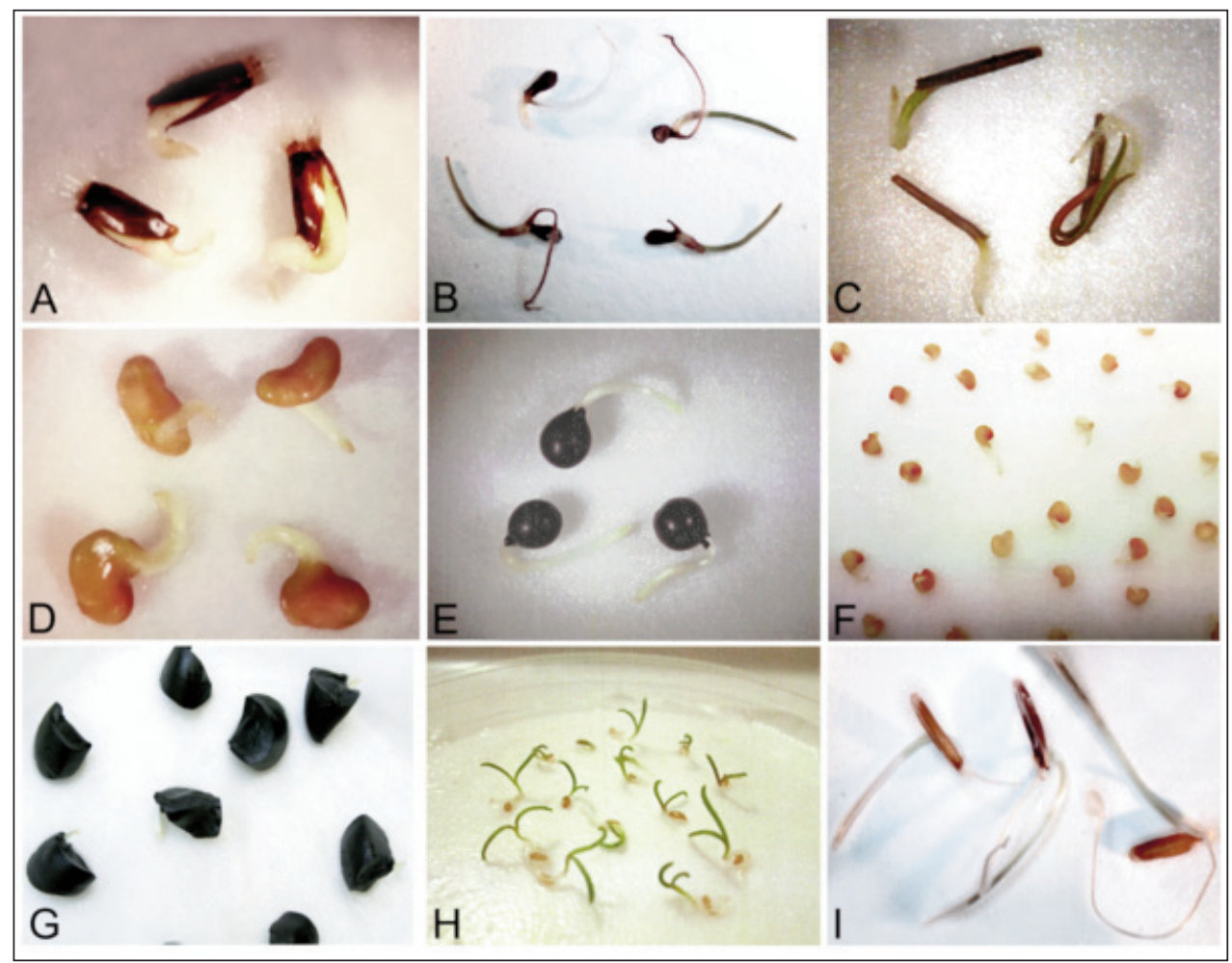

Fig. 1. Germinating seeds of: a, Centaurea sphaerocephala; b, Cyperus capitatus; c, Launaea fragilis; d, Medicago marina; e, Muscari gussonei; f, Ononis variegata; g, Pancratium maritimum; h, Plantago macrorrhyza; i, Thinopyron junceum.

temperature. However, our tests carried out in the same condition provided a final germination percentage of $53 \%$, while it reached $67 \%$ under full darkness. Other constant temperatures $\left(15^{\circ} \mathrm{C}\right.$ and $\left.25^{\circ} \mathrm{C}\right)$ yielded germination percentages not higher than $60 \%$, with lower values in the presence of light. Mavroeidi (2015) obtained high germination values in plants from Crete at constant temperature $\left(94.7 \%\right.$ at $10^{\circ} \mathrm{C}, 100 \%$ at $15^{\circ} \mathrm{C}$ and 98.7 at $20^{\circ} \mathrm{C}$ ) under full darkness; in these experiments, however, seeds were previously scarified (dispersal unit removed) and this clearly produced their higher and faster germination, because germination at $15^{\circ} \mathrm{C}$ decreased to $69 \%$ in seeds with pericarp.

\section{References}

Bacchetta, G., Fenu, G., Mattana, E., Meloni, F. \& Podda L. 2007: Conservazione ex situ e in situ della biodiversità vegetale dell'Area Marina Protetta di Capo Carbonara (Sardegna sud-orientale). - Fitosociologia 44(1): 157-164.

Balestri, E. \& Cinelli, F. 2004: Germination and early-seedling establishment capacity of Pancratium maritimum L. (Amaryllidaceae) on coastal dunes in the North-Western Mediterranean. - J. 
Coast. Res. 20(3): 761-770. https://doi.org/10.2112/1551-5036(2004)20 [761:GAEECO]2.0.CO;2

Ballesteros, D., Meloni, F. \& Bacchetta, G. (eds) 2015: Manual for the propagation of selected Mediterranean native plant species. - Cagliari.

Carta, A., Skourti, E., Mattana, E., Vandelook, F. \& Thanos, C. A. 2017: Photoinhibition of seed germination: occurrence, ecology and phylogeny. - Seed Sci. Res. 27(2): 131-153. https://doi.org/10.1017/S0960258517000137

Luciani, F., Cristaudo, A. \& Aricò, D. 2001: Germination ecology of three Plantago L. (Plantaginaceae) species living in a saline environment. - Pl. Biosyst. 135(2): 213-221. https://doi.org/10.1080/11263500112331350850

Mavroeidi, L. 2015: An ecophysiological study of seed germination and dormancy in eight native plant species of Crete and the design of a software application (introducing spatial information and visualization in the search engine) to improve the MAICh seed bank database. - Thesis (M.Sc.). Mediterranean Agronomic Institute of Chania.

Orsenigo, S., Montagnani, C., Fenu, G., Gargano, D., Peruzzi, L., Abeli, T., Alessandrini, A., Bacchetta, G., Bartolucci, F., Bovio, M., Brullo, C., Brullo, S., Carta, A., Castello, M., Cogoni, D., Conti, F., Domina, G., Foggi, B., Gennai, M., Gigante, D., Iberite, M., Lasen, C., Magrini, S., Perrino, E. V., Prosser, F., Santangelo, A., Selvaggi, A., Stinca, A., Vagge, I., Villani, M., Wagensommer, R. P., Wilhalm, T., Tartaglini, N., Duprè, E., Blasi, C. \& Rossi G. 2018: Red Listing plants under full national responsibility: extinction risks and threats in the vascular flora endemic to Italy. - Biol. Conserv. 224: 213-222.

Redondo-Gómez, S., Andrades-Moreno, L., Parra, R., Mateos-Naranjo, E. \& Sánchez-Lafuente, A. M. 2011: Factors influencing seed germination of Cyperus capitatus, inhabiting the moving sand dunes in southern Europe. - J. Arid Environ. 75(3): 309-312. https://doi.org/10.1016/j.jaridenv.2010.10.014

Royal Botanic Gardens Kew. 2019: Seed Information Database (SID). Version 7.1. Available from: http://data.kew.org/sid/ [Last Accessed July 2019].

Scippa, G. S. Petrollini, E., Trupiano, D., Rocco, M., Falco, G., Di Michele, M. \& Chiatante, D. 2011: Dormancy of Medicago marina (L.) seed. - Environ. Exp. Bot. 72: 320-329. https://doi.org/10.1016/j.envexpbot.2011.04.005

Address of the authors:

Cristina Salmeri ${ }^{1} \&$ Maria Trubia ${ }^{2}$,

${ }^{1}$ Department of Biological, Chemical and Pharmaceutical Sciences and Technologies (STEBICEF), University of Palermo, Via Archirafi 38, I 90123 Palermo, Italy. Email: cristinamaria.salmeri@unipa.it

${ }^{2}$ Seed Bank of spontaneous species, University of Catania (BGS-CT), Via Empedocle 58, 95128 Catania, Italy. 